\title{
Thyroid Hemiagenesis with Graves' Disease, Graves' Ophthalmopathy and Multinodular Goiter
}

\author{
Mehtap Cakir ${ }^{1}$, Sait Gonen ${ }^{1}$, Oguz Dikbas ${ }^{1}$ and Banu Ozturk ${ }^{2}$
}

\begin{abstract}
Thyroid hemiagenesis is a rare congenital anomaly in which one of the thyroid lobes with or without isthmus fails to develop. Here we present a woman patient with thyroid hemiagenesis, Graves' disease and ophthalmopathy with nodular goiter. Fine needle aspiration biopsy of the dominant nodule was suspicious of malignancy. The patient was referred for surgery for total thyroidectomy. Histopathological examination of the surgical material revealed benign features. The present case confirms that, although rare, a number of concomitant thyroid disorders can exist in a single patient with thyroid hemiagenesis just as it is seen for a normally developed thyroid gland.
\end{abstract}

Key words: thyroid hemiagenesis, autoimmune thyroid disease, goiter

(Inter Med 48: 1047-1049, 2009)

(DOI: 10.2169/internalmedicine.48.1984)

\section{Introduction}

Thyroid hemiagenesis where one thyroid lobe fails to develop, is a rare congenital abnormality which was first reported by Handfield-Jones in 1866 (1). Most of the patients with thyroid hemiagenesis are not diagnosed until a symptomatic or functional lesion occurs in the remaining single lobe. Thus, the total number of cases with thyroid hemiagenesis is uncertain. Here, we present a female patient who had hyperthyroidism and then was diagnosed to have thyroid hemiagenesis, Graves' ophthalmopathy and thyroid nodules.

\section{Case Report}

A 55-year-old woman presented to our endocrinology outpatient clinic with complaints of tiredness, palpitation, heat intolerance and periorbital puffiness. Three months earlier she had been started propylthiouracil $300 \mathrm{mg} / \mathrm{d}$ and propranolol $80 \mathrm{mg} / \mathrm{d}$ with a diagnosis of hyperthyroidism by a general practitioner. On past history, she had no history of thyroid disease or neck irradiation and had diabetes mellitus for 10 years. On physical examination she had a heart rate of $88 /$ minute, blood pressure of $130 / 70 \mathrm{mmHg}$ and a minimally palpable right thyroid lobe. There was no proptosis, extraocular eye movements were full, only upper eyelid retraction was noted in both eyes. When she was first seen by the general practitioner her thyroid function tests were as follows: fT3: $6.56 \mathrm{pmol} / \mathrm{L}$ (3.68-10.4), FT4:25.2 pmol/L (7.46-21.1), TSH: $0.05 \mathrm{mIU} / \mathrm{L}$ (0.34-5.60). After nearly 3 months of antithyroid drug treatment she was seen in our outpatient clinic and her TSH was found to be $0.14 \mathrm{mIU} / \mathrm{L}$ (0.58-1.64), fT3: $4.08 \mathrm{pmol} / \mathrm{L}$ (3.68-10.4) and fT4: 8.6 $\mathrm{pmol} / \mathrm{L}$ (7.46-21.1). Antithyroglobulin (Anti-Tg), antithyroperoxidase (anti-TPO) and TSH receptor antibodies (ZenTech s.a., Angleur, Belgium) were $2.2 \mathrm{IU} / \mathrm{mL}$ (0-4.9), 600 IU/mL (0-34) and $146 \mathrm{IU} / \mathrm{L}(0-10)$, respectively. On high resolution ultrasonography right thyroid lobe was $21 \times 23 \times 52$ $\mathrm{mm}$ in diameter, having a hypoechoic and heterogenous parenchyma with few hypoechoic nodules. Isthmus and the left lobe were undetectable. Dominant nodule was $12 \times 12 \times 7 \mathrm{~mm}$ in diameter located in medial part of the lobe (Fig. 1). The remaining nodules were $8 \times 9 \times 7 \mathrm{~mm}, 6 \times 6 \mathrm{~mm}$ and $5 \times 5 \mathrm{~mm}$ (inferoposterior) in diameter, respectively. Tc-99 $\mathrm{m}$ thyroid scan revealed increased uptake only in the right lobe with a hypoechoic region located on the medial aspect of the lobe, consistent with the location of dominant nodule detected on

${ }^{1}$ Division of Endocrinology and Metabolism, Selcuk University, Meram School of Medicine, Konya, Turkey and ${ }^{2}$ Department of Ophthalmology, Selcuk University, Meram School of Medicine, Konya, Turkey

Received for publication December 25, 2008; Accepted for publication March 9, 2009

Correspondence to Dr. Mehtap Cakir, cakirmehtap@yahoo.com 


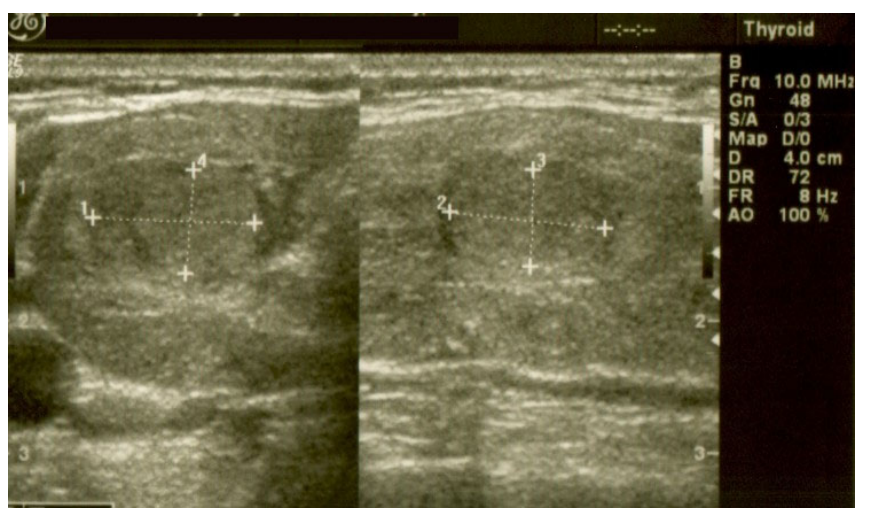

Figure 1. Ultrasonography of the dominant nodule in the right lobe.

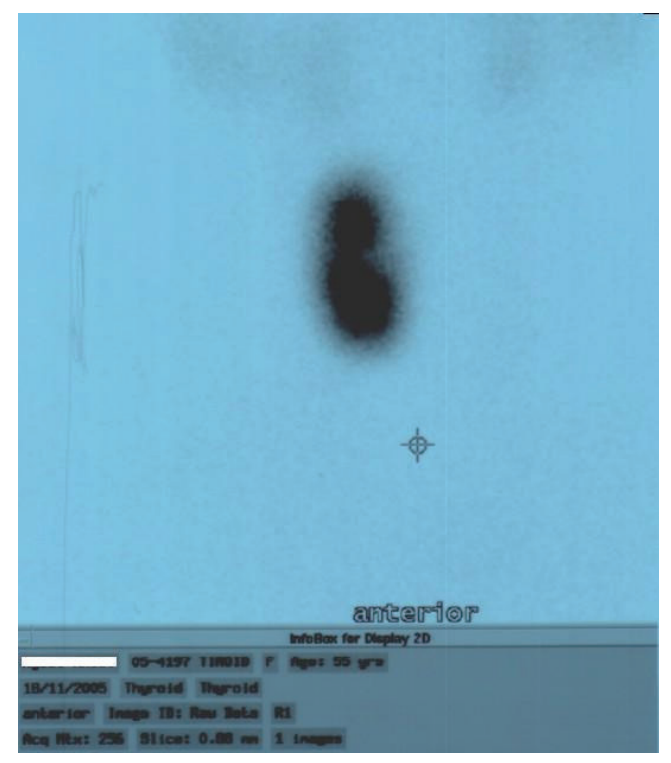

Figure 2. Tc- ${ }^{99} \mathrm{~m}$ thyroid scintigraphy showing uptake only in the right lobe and a hypoactive area in the medial aspect of the lobe.

ultrasonography (Fig. 2). There was no uptake in the left thyroid lobe. Based on these findings a diagnosis of thyroid hemiagenesis, Graves' disease and nodular goiter was made. Fine needle aspiration biopsy (FNAB) specimen of the dominant nodule was suspicious of malignancy and thyroidectomy was planned. However, the patient refused surgical intervention. Nearly two months after admission and under follow-up with oral antithyroid treatment, she developed new-onset increased lacrimation, burning and itching of eyes and increased periorbital puffiness. In ocular biomicroscopic examination of anterior segment, the conjunctiva was found to be hyperemic and chemotic, especially remarkable in the inferior quadrant. Ocular movements of the patient was recorded in 9 diagnostic positions of gaze and quantified using a grading system of (0-4) with 0 indicating full motion in that field of action and -4 indicating no motion. Lateral and medial gaze were restricted in both eyes, graded as -3 in the former and -1 in the latter, which warranted an orbital MRI. Exophthalmus was documented with Hertel exophthal-

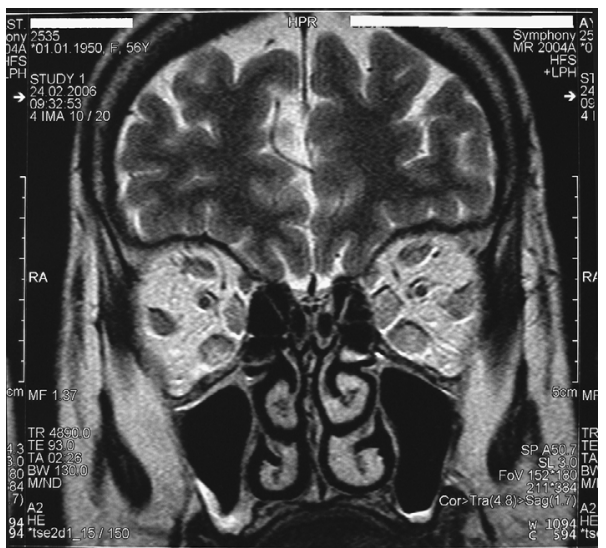

Figure 3. Hypertrophy of extraocular muscles on orbital MRI.

mometer readings of 18 in the right eye and 23 on the left eye. Lid retraction was also noted in the left eye, with the upper eyelid being $1 \mathrm{~mm}$ above limbus. Bilateral proptosis and hypertrophy of lateral, medial and inferior recti in both eyes were revealed in orbital MRI (Fig. 3). Regarding these findings the patient was graded as class 4 Graves' ophthalmopathy according to NO SPECS classification. Methylprednisolone $60 \mathrm{mg} /$ day PO was started. During her treatment with glucocorticoids, she accepted surgical intervention for the thyroid. Right lobectomy was performed. On pathological examination, thyroid parenchyma was found to be seperated by several fibrous bands and there were distinct nodularities. The nodules consisted of small and large follicles side by side. The follicles were surrounded by a single layer of epithelial cells. Some nodules were seperated from the surrounding tissue by fibrosis and in one nodule calcification was noted in the surrounding fibrous tissue. A moderate degree of lymphocytic infiltration was noted. In the dominant nodule follicular epithelium was of the Hürthle cell type. However, as no vascular or capsular invasion was seen, this nodule was reported as Hürthle cell adenoma. Postoperatively, her ophthalmopathy worsened under steroid treatment and orbital decompression surgery was performed. After surgery her eye symptoms and signs have nearly completely resolved except some degree of periorbital edema. She was rendered euthyroid with $150 \mu \mathrm{g}$ of oral L-thyroxine treatment.

\section{Discussion}

The prevalence of thyroid hemiagenesis ranges between $0.05 \%-0.2 \%$ in literature $(2,3)$. Thyroid hemiagenesis is seen with a female to male ratio of $3: 1$ and a left to right hemiagenesis ratio of $4: 1$. The isthmus is also absent in $50 \%$ of the patients (4). Coexisting thyroid disorders encountered with thyroid hemiagenesis are chronic lymphocytic thyroiditis, subacute thyroiditis, nodular goiter, primary or metastatic carcinoma and benign thyroid neoplasms with accompanying hyper-, hypo- or euthyroidism. However, thyroid hemiagenesis presenting with Graves' ophthalmopathy has 
been reported in only 2 cases previously $(5,6)$ and thyroid ultrasonography revealed an accompanying nodule in only one of these cases (6).

Genetic background is suspected in thyroid hemiagenesis as this rare disorder occurs in monozygotic twins, or together with other thyroid abnormalities within one family (7). In a review of inherited disorders of thyroid by Knobel and Medeiros-Neto, it was suggested that the genes most likely implicated in thyroid dysgenesis are those encoding transcription factors involved in the embryologic development of the thyroid (8). Thyroid-specific transcription factors TTF-1, TTF-2 and Pax- 8 are the most frequently encountered candidates in the etiology of thyroid dysgenesis (6), however no certain relationship has been demonstrated between thyroid hemiagenesis and mutations of these genes. Additionally, in an animal study it was shown that sonic hedgehog (Shh) knockout mice the thyroid primordium is correctly specified in the pharyngeal endoderm, but budding and dislocation were slightly delayed (9). In late develop- ment the thyroid failed to form a bilobed gland but instead a single thyroid mass was found unilaterally and mostly to the left of the midline.

In conclusion, congenital thyroid hemiagenesis cases are suspected by physical examination or unilateral uptake on thyroid scan. Conditions which cause functional hemiagenesis and are misdiagnosed with thyroid scan as congenital thyroid hemiagenesis are infitrative diseases such as amyloidosis, unilateral inflammation, toxic adenoma, primary and metastatic neoplasms (5). Thyroid ultrasonography is the gold standard method for confirming the diagnosis of thyroid hemiagenesis and has high sensitivity and specificity. Recognition of this rare congenital anomaly is important to avoid unnecessary surgery, contralateral neck exploration with its potential morbidity and radioactive iodine treatment. The present case confirms that, a number of concomitant thyroid disorders can exist in a single patient with thyroid hemiagenesis just as it is for a normally developed thyroid gland.

\section{References}

1. Handfield-Jones. Primary hyperparathyroidism with thyroid hemiagenesis. In: Handbuch der SystematischenAnatomie des Menschen. Henle J, Ed. Sohn: Friedrich Vlewig und Braunschweig, 1866: 538 (in German).

2. Shabana W, Delange F, Freson M, Osteaux M, De Schepper J. Prevelance of thyroid hemiagenesis: ultrasound screening in normal children. Eur J Pediatr 159: 456-458, 2000.

3. Maiorana R, Carta A, Floriddia G, et al. Thyroid hemiagenesis: Prevalance in normal children and effect on thyroid function. $\mathrm{J}$ Clin Endocrinol Metab 88: 1534-1536, 2003.

4. Melnick JC, Stemkowski PE. Thyroid hemiagenesis (hockey stick sign): A review of the world literature and a report of four cases. J Clin Endocrinol Metab 52: 247-251, 1981.

5. Ozgen AG, Saygili F, Kabalak T. Thyroid hemiagenesis associated with Graves' disease and Graves' ophthalmopathy: case report. Thyroid 14: 75-77, 2004.

6. McLean R, Howard N, Murray IP. Thyroid dysgenesis in monozygotic twins: variants identified by scintigraphy. Eur J Nucl Med 10: 346-348, 1985.

7. Baldini M, Orsatti A, Cantalamessa L. A singular case of Graves' disease in congenital thyroid hemiagenesis. Horm Res 63: 107110, 2005.

8. Knobel M, Medeiros-Neto G. An outline of inherited disorders in the thyroid hormone generating system. Thyroid 13: 771-801, 2003.

9. Fagman H, Grande M, Gritli-Linde A, Nilsson M. Genetic deletion of sonic hedgehog causes hemiagenesis and ectopic development of the thyroid in mouse. Am J Pathol 164: 1865-1872, 2004.

(C) 2009 The Japanese Society of Internal Medicine

http://www.naika.or.jp/imindex.html 